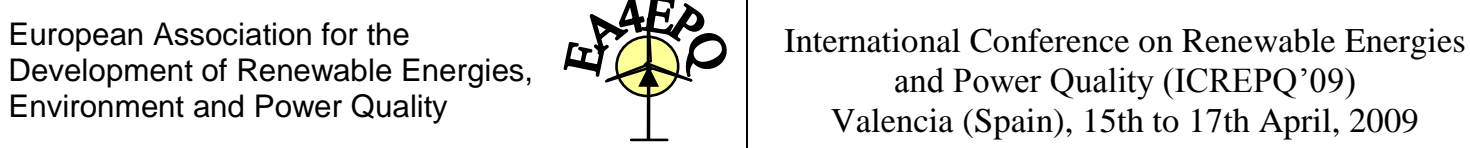

International Conference on Renewable Energies and Power Quality (ICREPQ'09)

Valencia (Spain), 15th to 17th April, 2009

\title{
Analysis of Electrical Field in a 6 kv Form-Wound Coil with Flat Shape Voids
}

\author{
C.H. Rojas, M.G. Melero, M. F. Cabanas, J.M. Cano, G.A. Orcajo, J. F. Pedrayes \\ ${ }^{1}$ Department of Electrical Engineering \\ University of Oviedo \\ Campus of Viesques, 33204 Gijon (Spain) \\ Phone/Fax number:+0034 985 182470, e-mail: chrojas@uniovi.es, melero@uniovi.es, \\ manes@uniovi.es , jmcano@uniovi.es, gonzalo@uniovi.es, pedrayes@uniovi.es,
}

\begin{abstract}
During the manufacturing process of form-wound coils small voids in the insulation groundwall may appear. These voids are usually caused by the presence of impurities, dirtiness, unsuitable pressure or temperature during pressing and curing processes, etc. The electrical stress into these voids may produce air ionization and thus partial discharge activity inside the insulation system. This phenomenon is one of main causes of the time life reduction in this type of winding. This paper presents a study of the electrical field distribution into a flat shape void placed at different positions of the insulation groudwall of a form wound coil. Several finite element models were developed in order to obtain the electric field distribution in the void when placed at different positions and orientation with respect to the electrical field direction.

Results presented in this paper are preliminary and belong to a wider study focused on the characterisation of the partial discharge pattern of the insulating system of form-wound coils after their manufacturing process.
\end{abstract}

Keywords Electric-field, form-wound coil, finite element, Partial discharge.

\section{Introduction}

The term electrical power quality brings together a series of technical concepts founded on an elementary basis: a continuous and uninterruptible power supply.

Ensuring the correct status of the insulating systems in electrical equipment is one of the primary goals to keep power supply without interruptions.

The presence of inclusions consisting of impurities, dirtiness, lack of compactness, etc, in the insulating system of medium and high voltage pieces of equipment may induce the formation of partial discharges that accelerate insulation ageing and thus may produce its final breakdown [1].

These inclusions have their origin in the manufacturing process or the normal degradation of the insulation system during its operation. Taking into account the relationship between the presence of voids in the insulation system and partial discharge activity it is clear that the analysis of the electrical field in a non perfect and heterogeneous dielectric may lead to a better knowledge about the conditions that may produce discharge activity. For this reason, a detailed study of the electrical field in the crossection of a medium voltage form-wound coil belonging to the stator winding of an asynchronous $6 \mathrm{kV}$ motor has been carried out. Different finite element models that include voids with different geometry and position inside the dielectric material have been developed. These models have been used to study the behaviour of the electrical field when the void position and orientation into the insulating system of the coil are modified.

\section{Study of the electrical field intensity distribution into the insulating system of a healthy form-wound coil}

In a first stage, the analysis of the distribution of the electrical field intensity in the crossection of a $6 \mathrm{kV}$ form-wound coil, belonging to the stator winding of an asynchronous machine, was carried out. This study was mainly focused on the calculation of the maximum values of field intensity in both, normal and tangential directions of a healthy coil including no voids or defects. The accurate location of the points of maximum electrical field intensity was previously done since their proximity to a void or inclusion of the insulating system may induce the formation of partial discharges. In a second stage, 18 finite element models of the coil with voids located at different positions and with different orientations with respect to the electrical field were developed. By means of these models, the influence caused by different factors such us the shape of the voids, their position, orientation etc. on the partial discharge generation has been studied. 


\section{A. 2D Finite element model of a form-wound coil}

As explained above, two dimension (XY plane) models of the crossection of a medium voltage stator winding coil were developed. All the components of the coil were accurately represented in the models. Figure 1 shows a photograph of the modeled coil. A simplified representation of the bi-dimensional models is shown in figure 2.

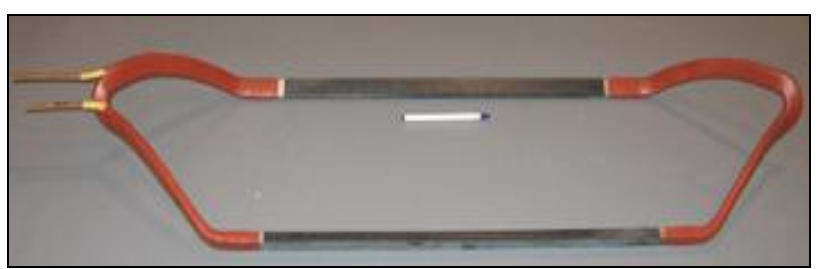

Fig. 1. 6kV form-wound modelled coil

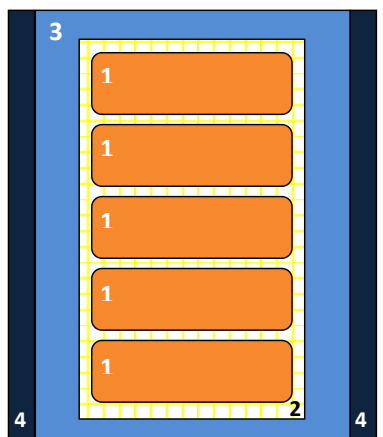

Fig. 2. Geometrical model of the crossection of the coil. (1: copper conductor, 2 conductor insulation, 3: groundwall insulation, 4: iron core)

Figure 3 shows a more detailed diagram of the crossection of coil. The different lines defined in the figure were used to calculate and represent the maximum values of electrical field intensity in the normal and tangential direction. Lines were located in the region of the conductor and groundwall insulating system since these areas are the most likely to present partial discharge activity related to manufacturing voids or defects. Table 1 shows the main data of the finite element models:

TABLE I.- Main data of the 2D FEM models

\begin{tabular}{|c|c|}
\hline Model & 2D \\
\hline Element Type & $\begin{array}{c}2^{\circ} \text { Order } \\
\text { Triangles }\end{array}$ \\
\hline Mesh dimension & 8000 Elements \\
\hline
\end{tabular}

\section{B. Models of a form-wound coil with insulation defects}

A study was carried out to analyze the effects caused on electrical field distribution by the presence of a flat void in different locations inside the insulating system of the coil. The position of the voids was selected to match with the lines defined in figure 3 . Figure 4 shows the sites where the voids where defined as well as its size and orientation. As shown in the figure, voids 1-3 correspond to defects on the conductor insulating system, while voids 4-6 are located inside the groundwall insulation.

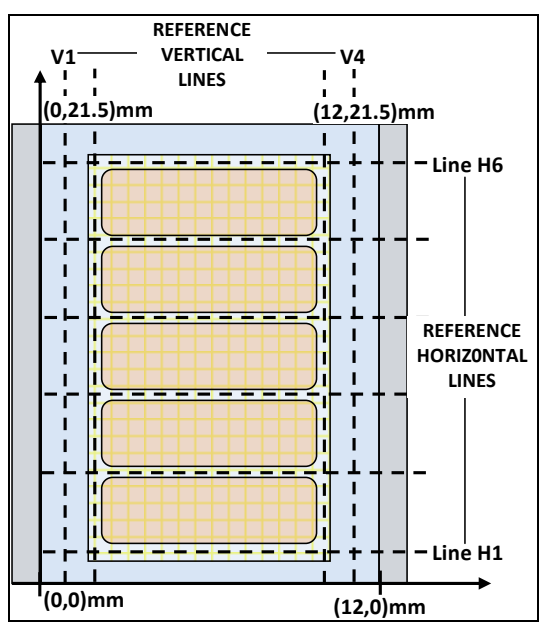

Fig. 3. Crossection of the coil (XY plane). Auxiliary lines for the study of the electrical field distribution

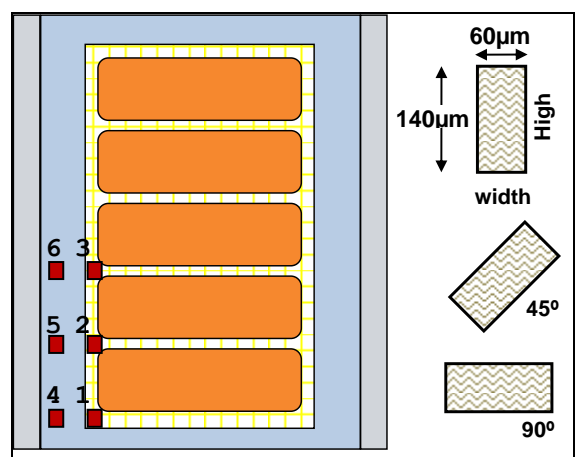

Fig. 4. Dimensions, location and orientation of the voids into the insulation systems (tree different orientations per void were studied).

The models are intended to identify the type of defect that produces the maximum concentration of electric field and, therefore, the higher partial discharge activity. Void dimensions were selected according to the magnitude of actual defects detected by manufacturers in industrial coils.

\section{B. Dielectrical breakdown inside the voids}

The breakdown characteristic of a void is a function of the product of the gas pressure into the void and its length. It is the so call Paschen's Law. The value of voltage that produces breakdown in the void can be estimated as the discharge voltage between equally spaced metal electrodes [2]:

$$
\mathrm{E}_{\mathrm{bd}}=\mathrm{p} \frac{\mathrm{B}}{\mathrm{C}+\ln \mathrm{p} \times \mathrm{d}}
$$

- $\quad \mathrm{E}_{\mathrm{bd}}$ being the dielectric breakdown voltage.

- $\quad p$ being the void gas pressure in Torr ( $\mathrm{mmHg}$ ),

- $\quad d$ being the distance between electrodes $(\mathrm{cm})$.

$\mathrm{B}$ and $\mathrm{C}$ are empirically calculated constants. $(\mathrm{B}=365$ Vcm-1 Torr-1 $\mathrm{C} \approx 1.18$ for the air). 
In the next figure, the breakdown characteristic (Paschen's Law) of the air is represented [1]. The dashed line shows the breakdown voltage value for the minimum size of simulated voids.

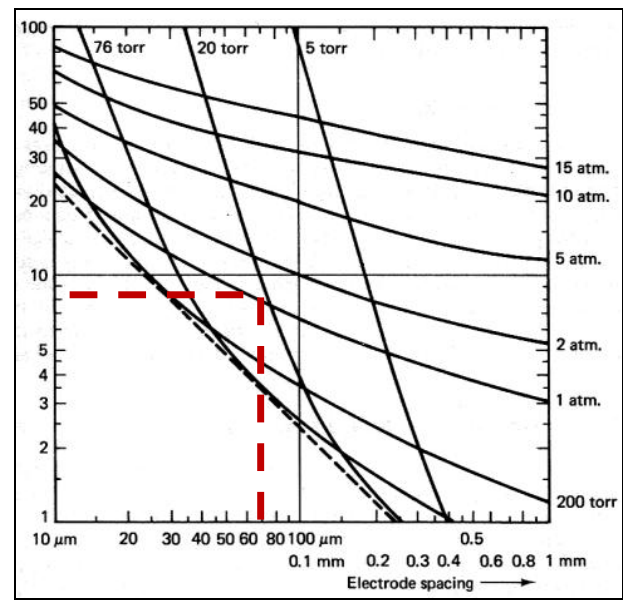

Fig. 5. Breakdown strength of the air $\left(\mathrm{E}_{\mathrm{bd}}\right)$ obtained from Paschen's Law formulation.

\section{Simulation results}

\section{A. Analysis of a healthy coil.}

A map of electrical field intensity in the crossecction of the coil is presented in the next figure. The maximum local field concentration takes places at "the corners" of the coil close to the top and bottom conductors.

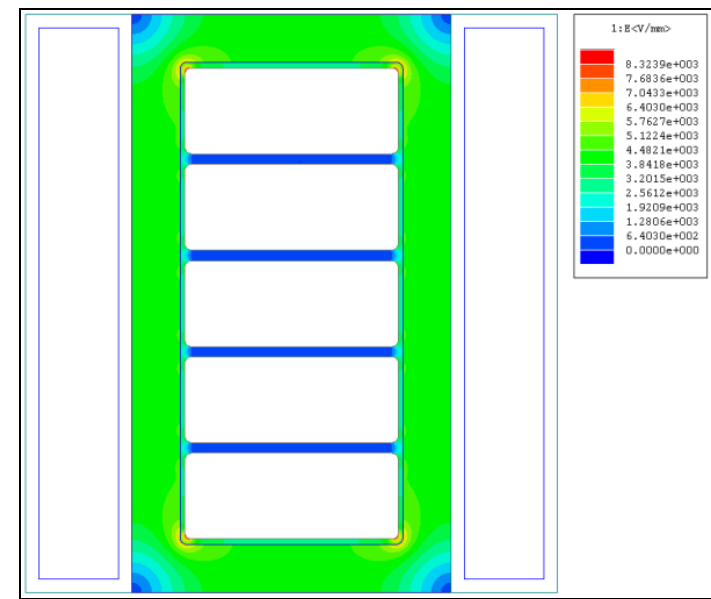

Fig. 6. Electrical field intensity distribution in the crossection of the coil

Figures 7 to 10 show the maximum value of $\mathbf{E}$ in the normal and tangential direction to the lines defined in figure 2. The direction of maximum electrical field intensity $\mathbf{E}$ into the coil coincides with the direction of maximum increase of the voltage $\mathbf{V}$, [3], as deduced from equation 1 :

$$
\mathrm{E}=\nabla \cdot \mathrm{V}
$$

The values obtained for $\mathbf{E}$ in all the positions and orientations of the void present a maximum of about 4.5 $\mathrm{kV} / \mathrm{mm}$. This value represents a $40 \%$ of the air breakdown voltage (see figure 5). However, as it will be shown below this value of the electrical field will be increased with the presence of the defect.

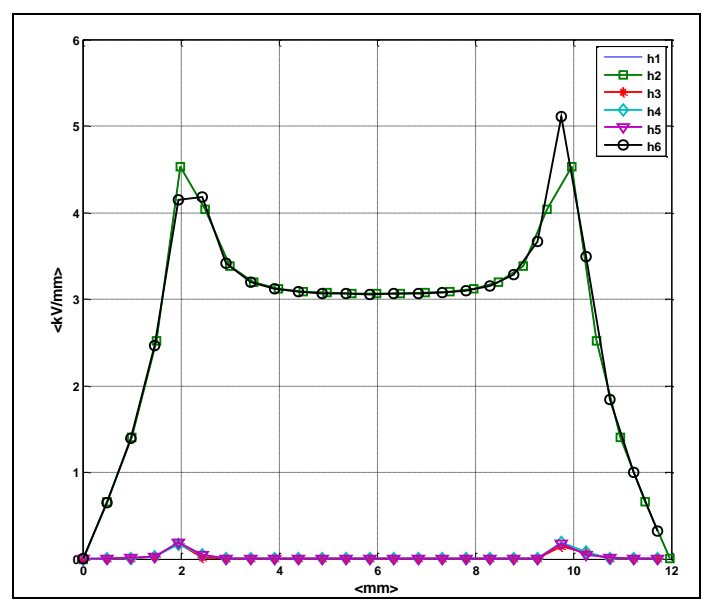

Fig. 7. Maximum value of the electrical field in the normal direction of the horizontal reference lines

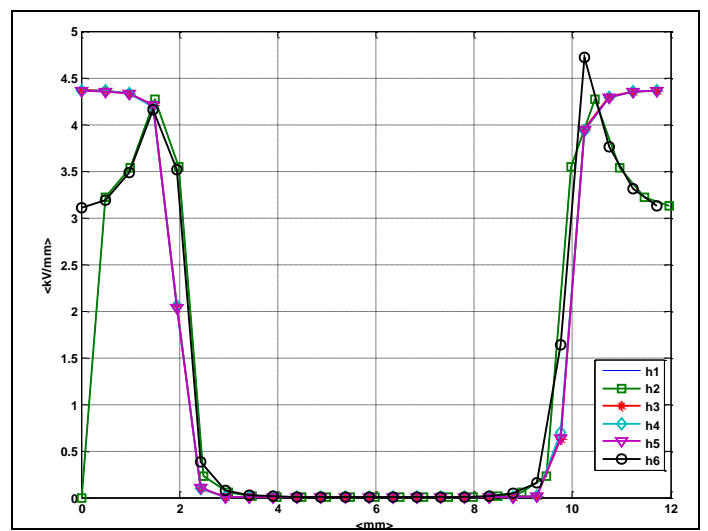

Fig. 8. Maximum value of the electrical field in the tangential direction of horizontal reference lines

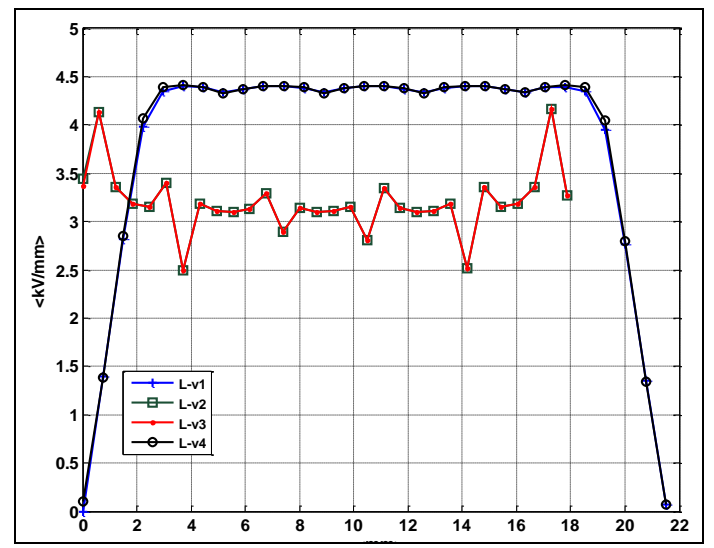

Fig. 9. Maximum value of the electrical field in the normal direction of vertical reference lines

B. Maximum values of potential difference across the voids

As explained above, a relationship between the position and orientation of voids and partial discharge activity exists. In fact, orientation and position of the void determine its maximum electrical field intensity. Simulation results showed that electrical field intensity 
across the void is the maximum when the void larger side is perpendicular to the field vector.

The maps of the distribution of maximum electric field intensity into the void placed at position 1 are shown in figure 11. As deduced from the map of colors obtained, in this case location of the void at $90^{\circ}$ presents the maximum electrical field intensity. As indicated above, in this location the larger side of the void is perpendicular to the electric field vector.

Partial discharge activity will start when the electrical stress in the void exceeds the breakdown dielectric limit. Values of breakdown voltage can be obtained from Paschen's curve for the air (figure 5).

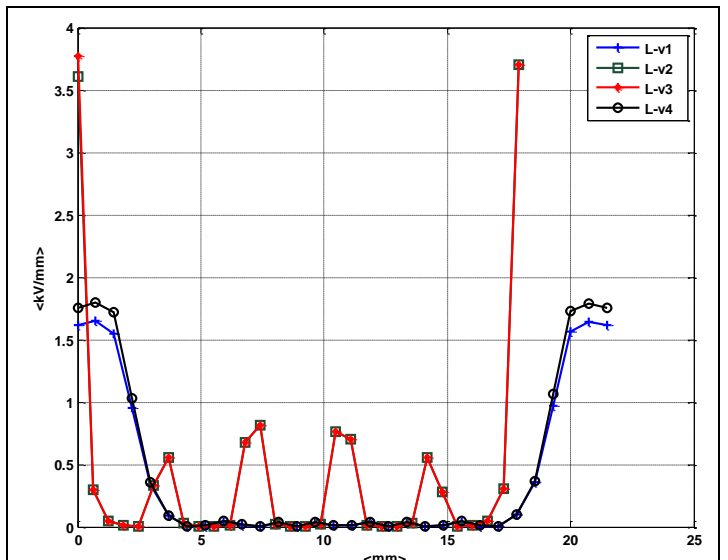

Fig. 10. Maximum value of the electrical field in the tangential direction of vertical reference lines

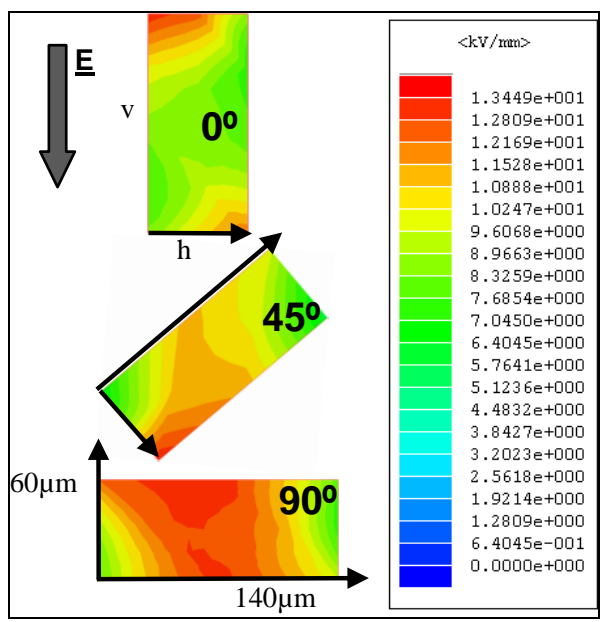

Fig. 11. Map of colors of the magnitude of field intensity inside the void located at position 1. Map obtained for different orientations of the void.

Figures 12 and 13 show the maximum values of $\mathbf{E}$ along the width and height of the void. Figure 14 shows with the same scale (minimum 4.5, maximum $13 \mathrm{kV} / \mathrm{mm}$ ) the color map of electrical field intensity in the voids located in positions 1 to 6 (Fig. 4.). According to the obtained results voids placed between conductors show a lower value for electrical field intensity, therefore, they are not likely to produce partial discharge activity.

Voids in position 1 present the highest field intensity with a value of $13.2 \mathrm{kV} / \mathrm{mm}$. Voids sited inside the groundwall and close to the magnetic core also showed a relatively high value for electrical field intensity.

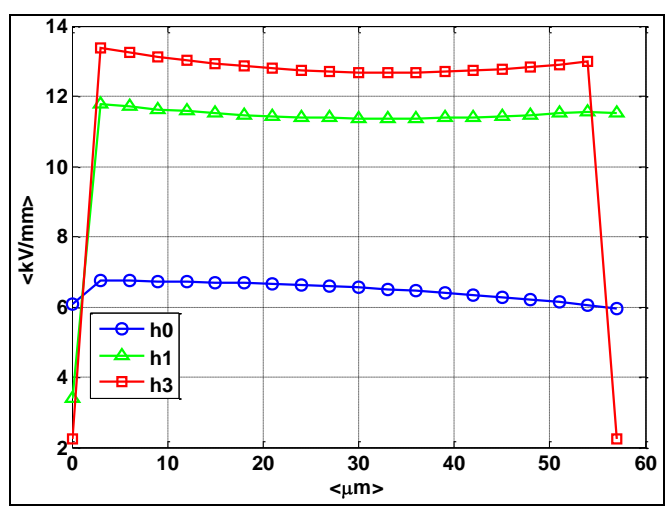

Fig. 12. Maximum values of $\mathbf{E}$ across the width of void placed in position 1: 0 : vertical orientation; $1: 45^{\circ}$ and 2: horizontal position

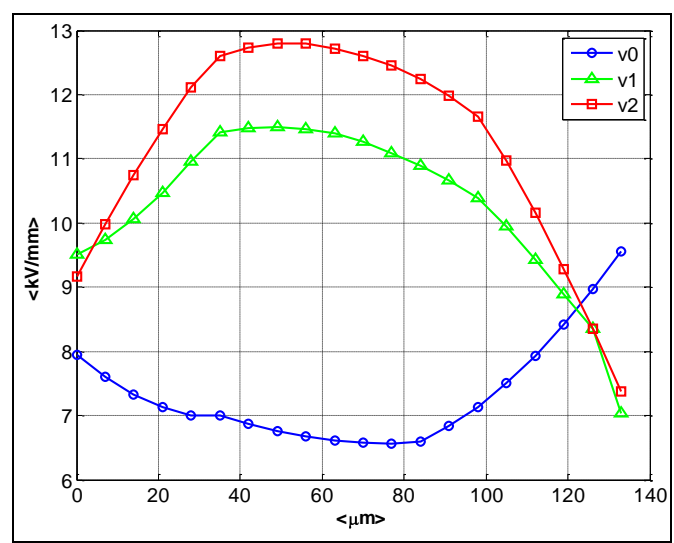

Fig. 13. Maximum values of $\mathbf{E}$ across the high of void placed in position 1: 0 : vertical orientation; $1: 45^{\circ}$ and 2 : horizontal position

Figure 15 shows several maps of color for the electrical field intensity obtained for the 6 positions of the void with their initial orientations $\left(90^{\circ}\right.$ for the void in position 1 and $0^{\circ}$ for the rest of the defects). In this figure one can appreciate how the maximum values for the electrical field correspond to the void in position 1 which presents its larger side perpendicular to the direction of the electrical field. From the view of this graph it can be concluded that maximum field concentration always happens when the void is oriented in such a way that its larger side is normal to the electrical field lines, i.e. $90^{\circ}$ for the void in position 1 and $0^{\circ}$ for the rest of the locations.

Figures 15 and 16 show electrical field intensity along the width and height of the voids located in the 6 studied positions. All the results presented in these figures have been obtained for the orientation that produces the maximum electrical field intensity.

Once the above results were obtained, the following stage in the study consisted of analyzing which of the studied defects were able to produce the electrical breakdown. To achieve it Paschen curve presented in figure 5 was used. By means of this graph it was checked that voids in positions 2 and 3 never surpassed the air breakdown limit in any of their possible orientations $\left(0^{\circ}, 45^{\circ}\right.$ and $\left.90^{\circ}\right)$. However, voids placed in position 1 presented a 
maximum value for the electrical field higher than the breakdown limit (around $11 \mathrm{kV} / \mathrm{mm}$ ). The rest of the studied defects produced maximum values of voltage very close to the breakdown limit.

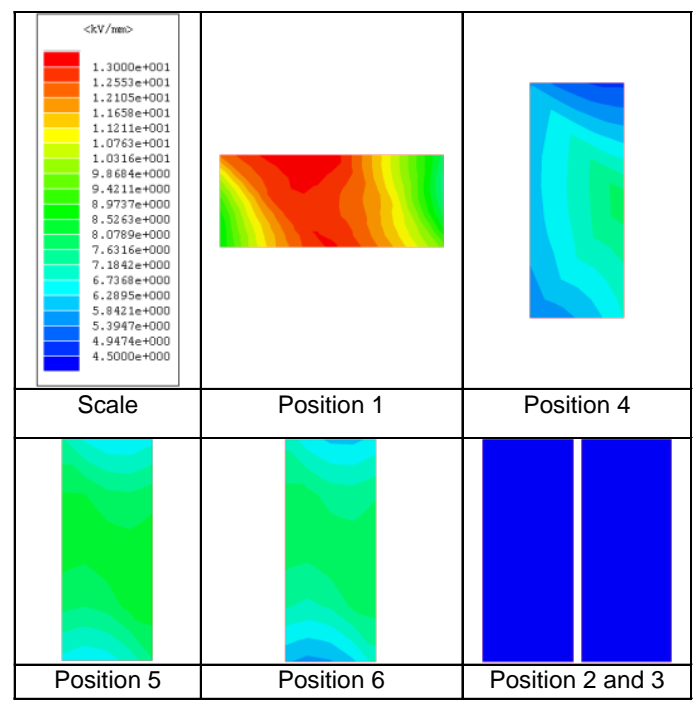

Fig. 14. Maps of the Intensity of the electric field inside the voids placed in positions $1 \rightarrow 6$ (Fig. 4).

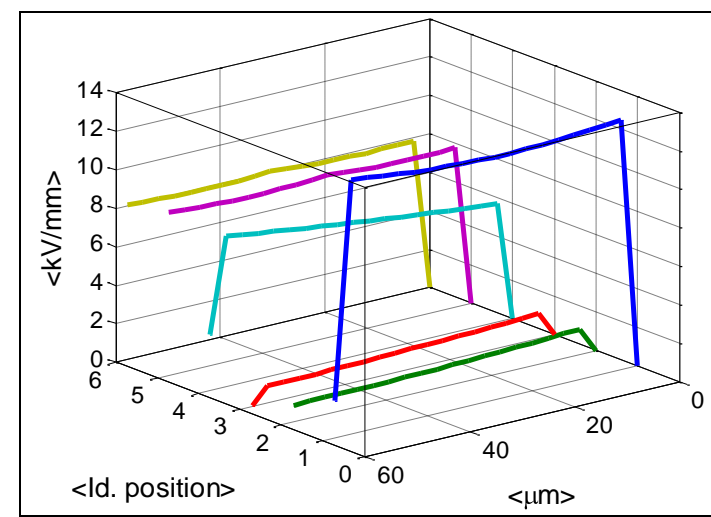

Fig. 15. Absolute maximum values of $\mathbf{E}$ across the width of voids located at positions 1-6 for all the studied orientations.

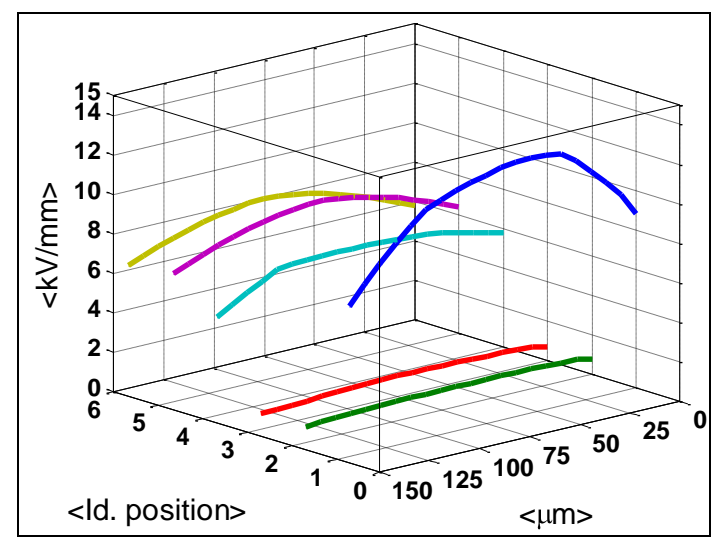

Fig. 16. Absolute maximum values of $\mathbf{E}$ across the height of void located at position 1-6 for all the studied orientations

The graphs presented in figures 17 and 18 allowed a perfect analysis of the influence caused by the void orientation on the maximum electrical field intensity. As explained above, all the obtained results demonstrated that maximum field intensity is produced when the flat shape void is located in a horizontal position, or what is the same, its long side is perpendicular to electric field. This fact can be observed in figures 17 and 18 that show how the maximum values of $\mathrm{E}$ are produced for the void in position 1 when its orientation is $90^{\circ}$ and for the voids in positions 2 to 6 when its orientation is $0^{\circ}$. Therefore, in all the cases the maximum values are generated when the electric field vector $\overrightarrow{\mathbf{E}}$ and the largest dimension of the void are perpendicular each other.

In positions 4,5 and 6 the effect caused by modifying the orientation of the void was a clear increase of about $3 \mathrm{kV} / \mathrm{mm}$ in the value of the electrical field. In these three cases field values were close to the breakdown limit.

In the case of the defect located in position 1 the void orientation presented the maximum influence in the electrical field distribution. For this position, the values of maximum field oscillated between 7 and $13 \mathrm{kV} / \mathrm{mm}$ depending on void orientation. Therefore, it can be concluded that partial discharge activity in the defect will strongly depend on void orientation.

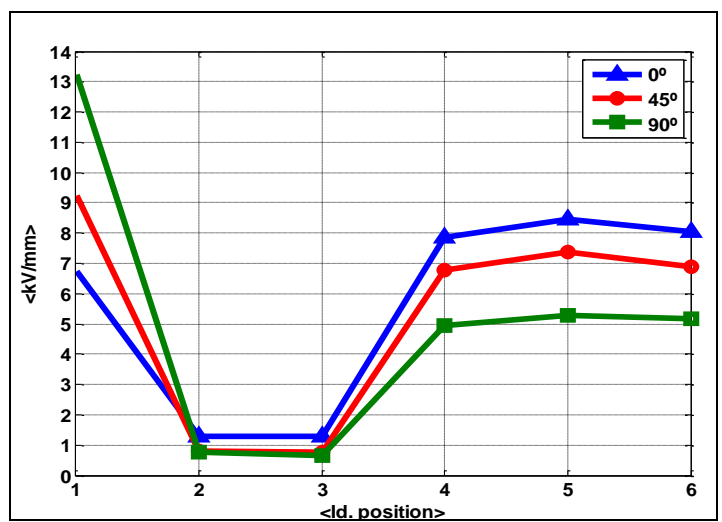

Fig. 17. Maximum values of $\mathbf{E}$ into of void situated at positions 16. Values calculated along the width of void

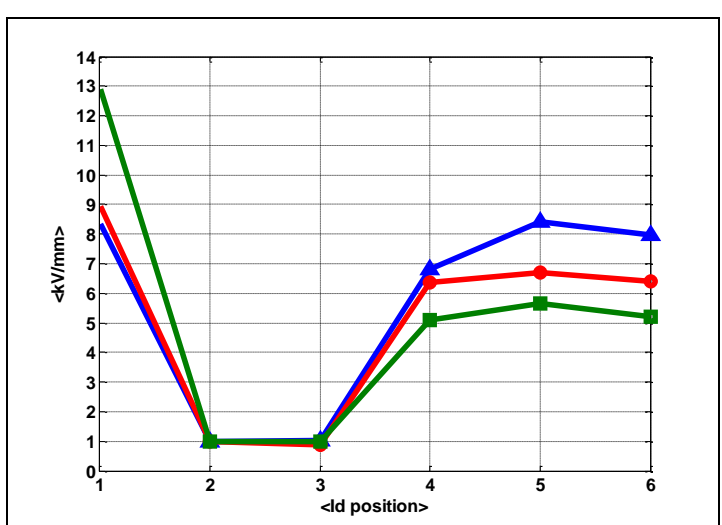

Fig. 18. Maximum values of $\mathbf{E}$ into of void situated at positions 16 of insulation. Value calculated along the height of void

Table II summarizes the results presenting the maximum value of E obtained for the 6 studied positions. Together with the calculated values the orientation at what they were simulated is also indicated. 
TABLE II. Maximum values of $E$ for the voids in the 6 different positions and orientation that produced it

\begin{tabular}{|c|c|c|}
\hline Position & $\begin{array}{c}\text { Maximun } \\
\text { value of E }\end{array}$ & $\begin{array}{c}\text { Orientation of } \\
\text { the void }\end{array}$ \\
\hline 1 & $13.2 \mathrm{kV} / \mathrm{mm}$ & $90^{\mathbf{o}}$ \\
\hline 2 & $1.3 \mathrm{kV} / \mathrm{mm}$ & $0^{\mathbf{o}}$ \\
\hline 3 & $1.2 \mathrm{kV} / \mathrm{mm}$ & $0^{\mathbf{o}}$ \\
\hline 4 & $7.9 \mathrm{kV} / \mathrm{mm}$ & $0^{\mathbf{o}}$ \\
\hline 5 & $8.4 \mathrm{kV} / \mathrm{mm}$ & $0^{\mathbf{o}}$ \\
\hline 6 & $8.1 \mathrm{kV} / \mathrm{mm}$ & $0^{\mathbf{o}}$ \\
\hline
\end{tabular}

The difference of potential across the void (from one end to the other) can be calculated from the values obtained for the electrical field, [3]:

$$
\mathrm{V}=\int_{\mathrm{a}}^{\mathrm{b}} \mathrm{E} \cdot \mathrm{dl}
$$

E being the electrical field vector, dl a differential length of an imaginary line ( $\mathbf{L})$ through the width or height of the cavity and $\mathbf{a}, \mathbf{b}$ the two endpoints of $\mathbf{L}$.

TABLE III. End to end maximum voltage values in the insulation across the width and height of the void

\begin{tabular}{|c|c|c|}
\hline $\begin{array}{c}\text { Void } \\
\text { Position }\end{array}$ & $\begin{array}{c}\text { Width } \\
\text { direction }\end{array}$ & $\begin{array}{c}\text { Height } \\
\text { direction }\end{array}$ \\
\hline 1 & $733.8 \mathrm{~V}$ & $1618.3 \mathrm{~V}$ \\
\hline 2 & $61.0 \mathrm{~V}$ & $130.8 \mathrm{~V}$ \\
\hline 3 & $60.8 \mathrm{~V}$ & $128.7 \mathrm{~V}$ \\
\hline 4 & $410.6 \mathrm{~V}$ & $925.9 \mathrm{~V}$ \\
\hline 5 & $486.2 \mathrm{~V}$ & $1058.9 \mathrm{~V}$ \\
\hline 6 & $740.9 \mathrm{~V}$ & $996.7 \mathrm{~V}$ \\
\hline
\end{tabular}

\section{Conclusions}

The electrical field distribution in an industrial formwound medium voltage coil including some physical defects that may lead to a complete breakdown has been studied by means of finite element models.

Models were designed including in the crossection of the coil a flat shape void placed in different positions and angular orientations. By means of these models electrical field across the void was calculated in all the cases.

The results obtained allowed us to confirm that maximum values of electric field are obtained when the void orientation presents the larger length of the void perpendicular to the electric field lines.
When electric field values are close to the air dielectric breakdown limit, void orientation determines whether the breakdown and thus the partial discharge occur or not.

Regarding voids position it was observed that maximum electrical field values were obtained for the void located in the corner of the coil close to the top and bottom conductors (position 1 in the study).

Location and orientation of the void will influence the pattern of evolution of electric charge distribution during coil operation. The analysis and comprehension of this pattern is essential for a correct evaluation of the insulating system, especially when manufacturing defects are trying to be detected.

\section{References}

[1] F.H. Kreuger. "Partial Dischrage detection in HighVoltage Equipment. Edt Butterworths, London 1989.

[2] G. Raju "Dielectrics in Electric Fields". Edt. Marceld Ekkeirn,C . NY, 2003

[3] W.H. Hayt. "Engineering Electromagnetics". Edit. Tata McGraw-Hill. Indian 2006.

[4] S. Zamora Sorí, M. C. Caminero, M. G. Melero, M. F. Cabanas, C. H. Rojas, G. A. Orcajo. "Diagnosis of failures in epoxy-mica insulating systems by means of partial discharges analysis and finite element Models". Vol 1, pp. 162-167. Symposium on Diagnostics for Electric Machines, Power Electronics and Drives SDEMPED 2003. Atlanta, GA, USA 24-26 August 2003.

[5] G.C. Stone. "Partial Discharge Diagnostics and Electrical Equipment Insulation Condition Assessment". IEEE Transactions on Dielectrics and Electrical Insulation Vol. 12, No. 5; October 2005.

[6] R. Bartnikas. "Partial Discharges Their Mechanism, Detection and Measurement". IEEE Transactions on Dielectrics and Electrical Insulation Vol. 9 No. 5, October 2002.

[7] P. Morshuis "Degradation of Solid Dielectrics due to Internal Partial Discharge: Some thoughts on progress made and where to go now". IEEE Transactions on Dielectrics and Electrical Insulation Vol. 12, No. 5; October 2005.

[8] Jim Lux "High Voltage Experimenter's Handbook". 1998

[9] Opera "Reference manual". Edt. Vector Field Limited, Oxford, 1999. 